\title{
CRISPR as a Potential Technique to Reduce Suicide Risk
}

\author{
Hidemi Zamora ${ }^{1}$ and Javier Cornejo" \\ ${ }^{1}$ San Ignacio de Recalde School, San Borja, Peru \\ \#Advisor
}

\section{$\underline{\text { ABSTRACT }}$}

As suicide is the nineteenth leading cause of death worldwide, it is important to focus on discovering ways to reduce the risk of suicide-related death as much as possible. With CRISPR starting to become increasingly popular over the past few years, this gene editing technique has been used to study how to edit, turn off, or knock out multiple parts of the genome. However, research on genes related to diseases as cystic fibrosis or Alzheimer's disease has been mainly prioritized and, even though they are of high importance as well, important issues such as suicide have been left into oblivion. Four genes have been proven to be key in influencing suicide risk, showing that not only environmental factors account for an increased possibility of death by this cause. Therefore, gene editing techniques such as CRISPR could be applied in order to knock out those genes and reduce this risk. This research used Synthego's guide RNA design tool to predict how the use of CRISPR can be helpful in knocking out those four suicide-related genes and, consequently, in preventing suicide. The top-ranked guide RNAs for each gene were used, showing the best results possible and with the least number of off-targets, which, in turn, demonstrates the effectiveness of CRISPR as a potential technique to reduce the number of suicide-related deaths worldwide.

\section{Introduction}

In 2019, suicide was considered as the nineteenth leading cause of death worldwide, with over 700,000 deaths per year (World Health Organization, 2021). Even though environmental factors such as prolonged stress, access to drugs and firearms, social isolation, traumatic life events, exposure to someone else's suicide, among others may increase the risk (American Foundation for Suicide Prevention, 2019), suicide is a multifactorial behavioral phenotype that has been proven to be significantly affected by genetic factors as well since it has a heritability of between 40 and 50 percent (McGuffin et al., 2001). Therefore, due to the fact that suicide rates have had in increase of 33 percent between 1999 and 2019 (Centers for Disease Control and Prevention, 2021), multiple studies focusing on how someone's genotype may affect the risk of them having a suicide-related death have arisen.

Furthermore, the use of CRISPR (clustered regularly interspaced palindromic repeats) has been significantly increasing over the past few years. This gene editing technique allows scientists to correct, knock out, or turn on genes in cells and organisms quickly, easily, and cheaply. In fact, this is a natural process that takes place as part of the immune system of bacteria to defend themselves against viruses. However, scientists have been able to recreate it in laboratories, allowing them to edit any organism's genome. To perform CRISPR, it is necessary to have a guide RNA (gRNA) that matches the desired gene, as well as Cas9, which stands for CRISPR-associated protein. This endonuclease causes double stranded DNA to break and, by adding a separate DNA sequence to the test tube, homology directed repair can take place so that the molecule can be reconstructed using that added sequence as a template. This allows scientists to modify any part of the genome, or even just activate or inactivate a specific gene (Redman et al., 2016). 
In the specific case of knocking out a gene with CRISPR, dsDNA is broken by Streptococcus pyogenes Cas9 (SpCas9). Afterwards, as non-homologous end joining is prone to making errors, the attempt to fix the broken molecule usually ends up generating indels which, in turn, cause frameshift mutations that would make the gene no longer able to be transcribed and translated adequately (Doench, 2017). It is important to make clear that indels make reference to the insertion or deletion of bases somewhere in the genome, affecting the reading frame, that is, the grouping of nucleotides into codons, each of which will then be transcribed and translated into amino acids (Nowicki, 2017).

When selecting the cut site in CRISPR to knock out a gene, sites that code for amino acids near the N' terminus or the C' terminus of the protein are usually avoided. This is due to the fact that, in the case of the $\mathrm{N}$ ' terminus (free amine end of the protein chain), the cell could use an alternative ATG sequence (start codon) and, in the case of the C' terminus (free carboxylic acid end), the chances of creating a non-functional allele could decrease if the cut is done there (Doench, 2017).

CRISPR's increasing popularity has made many scientists research on which genetic disorders, or traits in general, can be prevented, cured, or just changed by editing the genome with this technique. Despite the current main focus being diseases like cystic fibrosis, Duchenne's muscular dystrophy, cancer, and Alzheimer's disease (Redman et al., 2016), suicide risk could be reduced as well by using gene editing techniques such as CRISPR since there are multiple genetic factors that have a huge impact on it.

This paper will discuss the genes that have been proven to play an important role in suicide risk, as well as how this discovery can be used to potentially implement CRISPR as a way to knock out those DNA segments and, in turn, reduce the number of suicide-related deaths.

\section{Methods}

This paper compiles studies regarding genes that have been shown to increase the risk of suicide-related death by analyzing their impact on high-risk extended families. The literary review was carried out by searching in systematic bibliographic databases, as well as in the references of relevant publications.

Additionally, Synthego's guide RNA design tool was used to predict how the knockout of genes APH1B, AGBL2, SUCLA2, and SP110 could be used as a potential technique to reduce suicide risk in patients who have unfavorable alleles. This tool is of great help when studying gene editing techniques, especially CRISPR, as it not only shows all genes of the human genome, but also the complete set of genes of Drosophila melanogaster, Mus musculus, and many other species that are commonly used in laboratory experiments. Moreover, it chooses the primary sequence, selects the early coding region, identifies potential targets, computes off targets against 304,792,603 sites, calculates the Azimuth score, and picks the top-ranked guide RNAs to knock out the selected gene. This allows scientists and researchers to be able to choose the most convenient gRNA when studying CRISPR so that the best results possible can be achieved.

\section{Literature Review}

Even though suicide risk can be increased by psychological, sociological, economic, and environmental factors, a person's genotype may make them more likely to have a suicide-related death. In order to prove this point, multiple studies focusing on this topic have been made. One of them showed, by analyzing monozygotic and dizygotic twins, that monozygotic ones have a notably higher concordance rate for both attempted (failed) and completed suicide in comparison to dizygotic twins (Dwivedi, 2012). A large Australian twin study had similar findings, showing that there was a 17-fold increase in the risk of attempting suicide in the case of monozygotic twins when the co-twin had previously made a serious suicide attempt (Pedersen \& Fiske, 2010). Additionally, it was proven by adoption studies that an adoptee with parents who had died by suicide was six times more likely to have a suicide-related death in comparison to adoptees with suicidal adoptive parents, suggesting that genetics play an important role in the probability of 
having a suicide-related death (Bondy et al., 2006). Later, a study compiling all these data was able to estimate the impact of genetics on suicide risk at around 43 percent, with the remaining 57 percent accounted for by environmental factors (McGuffin et al., 2001).

A study made by Coon et al. (2020) analyzed 43 extended families of between seven and nine generations who had a high risk of dying by suicide in order to find the genes involved in that risk. They used distantly related, high-risk cases in order to minimize shared environmental effects and magnify genetic ones, as well as enrich for genetic homogeneity, so that the most accurate results possible could be achieved and not confused with the effects the environment could have had in the studied subjects. In addition, the sample was taken from population-wide ascertainment, meaning that none of the participants had a specific psychiatric diagnosis.

From the results of this study, it was concluded that, as the suicide cases in those high-risk families occurred mostly at a young age, it is most likely that familial genetic risks play a way more important role than environmental factors in suicide-related deaths of young people since there is still a limited accumulation of the effects caused by the environment at that age. This conclusion was also supported by a sample of around 1300 genotyped cases, which matched the aforementioned discovery.

Moreover, after the use of the statistical method Shared Genomic Segments (SGS), 207 genes were implicated for targeted follow-up. This led to the discovery of eighteen genes that could probably be linked to suicide risk. Among those, fifteen had already been associated with inflammation, proving previous evidence of the relationship between suicide risk and inflammatory conditions. In addition, around 1300 Utah completed suicide cases were studied, resulting in the discovery of four genes proven to be associated with suicide: APH1B, AGBL2, SP110, and SUCLA2.

APH1B is a gene that codes for a multi-pass transmembrane protein part of the gamma-secretase complex. That protein helps in stabilizing the presenilin holoprotein in that multi-subunit protease complex and in catalyzing the cleavage of integral proteins, including the beta-amyloid precursor protein and notch receptors. It has also been associated with risk of getting Parkinson's and Alzheimer's diseases (Bekris et al., 2015). AGBL2 is a gene that codes for an ATP/GTP binding protein that plays an important role in brain function and structure (Karaca et al., 2015). SP110 is a gene that codes for a protein that is part of a leukocyte-specific nuclear body protein complex. It has been suggested that it may influence gene transcription, as well as immunodeficiency and pathogen resistance as it is involved in regulating cell division, apoptosis, and the normal functioning of the immune system. As it is related to inflammation and immune risk, it proves the idea of suicide risk increasing by inflammatory conditions (Leu et al., 2018). Finally, SUCLA2 is a gene that codes for a mitochondrial tricarboxylic acid cycle protein that plays an important role in supplying energy to synapses in the nervous system. It has been related to hypoxia and suicide risk as well (Völgyi et al., 2015). In fact, these four genes found to be suicide-related did not overlap with any specific patterns of psychopathology, showing their direct relationship with suicide risk exclusively.

\section{Findings}

Using Synthego's guide RNA design tool, models of how CRISPR could be used to knockout all four genes proven to be suicide-related were made.

In the case of the APH1B gene, which has a transcript Ensembl identifier of ENST00000261879, the topranked guide RNA had the sequence UCUUGCCAUGAACCAAACAA, which targeted the sequence AACAAACCAAGTACCGTTCT, located in the fifteenth human chromosome. This target sequence is located towards the start of the gene's coding sequence, plus it is located in an exon that results from most transcripts, making this guide RNA the most convenient possible for knocking out this gene. As shown in Figure 1, here the Cas9 ribonucleoprotein will bind to the antisense strand of the selected gene, generating a double-stranded break at the 63,279,202 location and having GGG as its PAM (protospacer adjacent motif) region. 


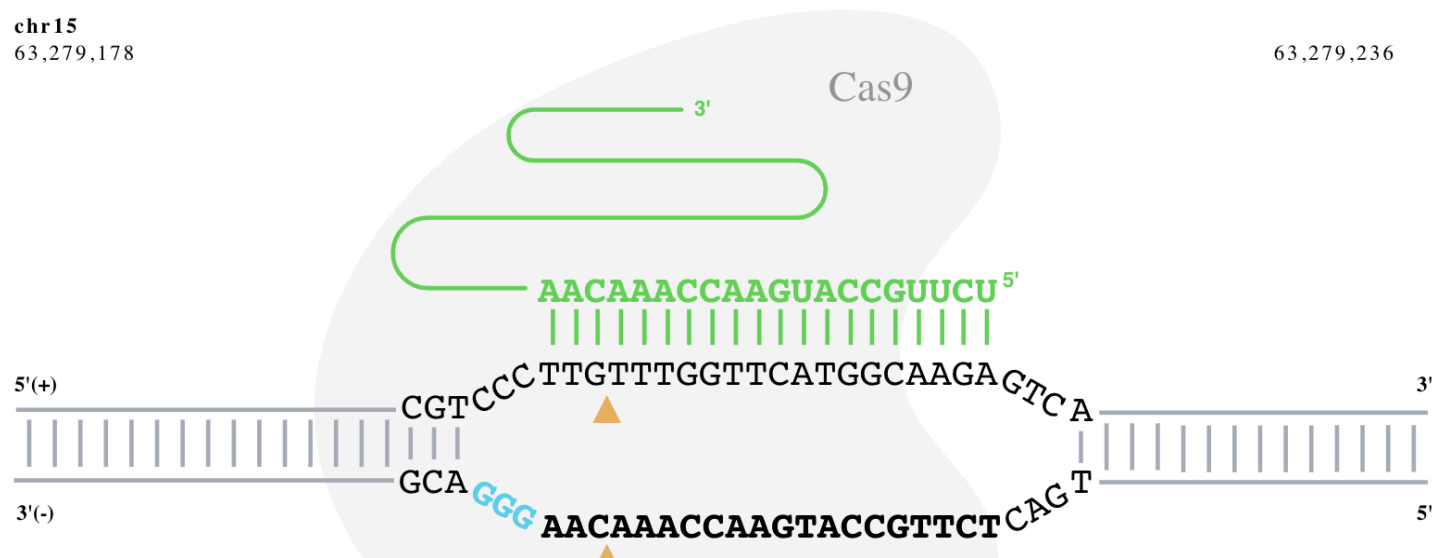

Figure 1. Top-ranked guide RNA sequence (shown in green) for the APH1B gene. Nucleotide sequence in light blue represents the PAM region. Orange triangles show the cut site. DNA segment in bold represents the target sequence (Synthego, 2021a).

In the case of the AGBL2 gene, which has a transcript Ensembl identifier of ENST00000525123, the topranked guide RNA had the sequence UGCCAAUUUUUAGCUCAGAG, which targeted the sequence GAGACTCGATTTTTAACCGT, located in the eleventh human chromosome. This target sequence is located towards the start of the gene's coding sequence, plus it is located in an exon that results from most transcripts, making this guide RNA the most convenient possible for knocking out this gene. As shown in Figure 2, here the Cas9 ribonucleoprotein will bind to the sense strand of the selected gene, generating a double-stranded break at the 47,710,508 location and having GGA as its PAM region. 


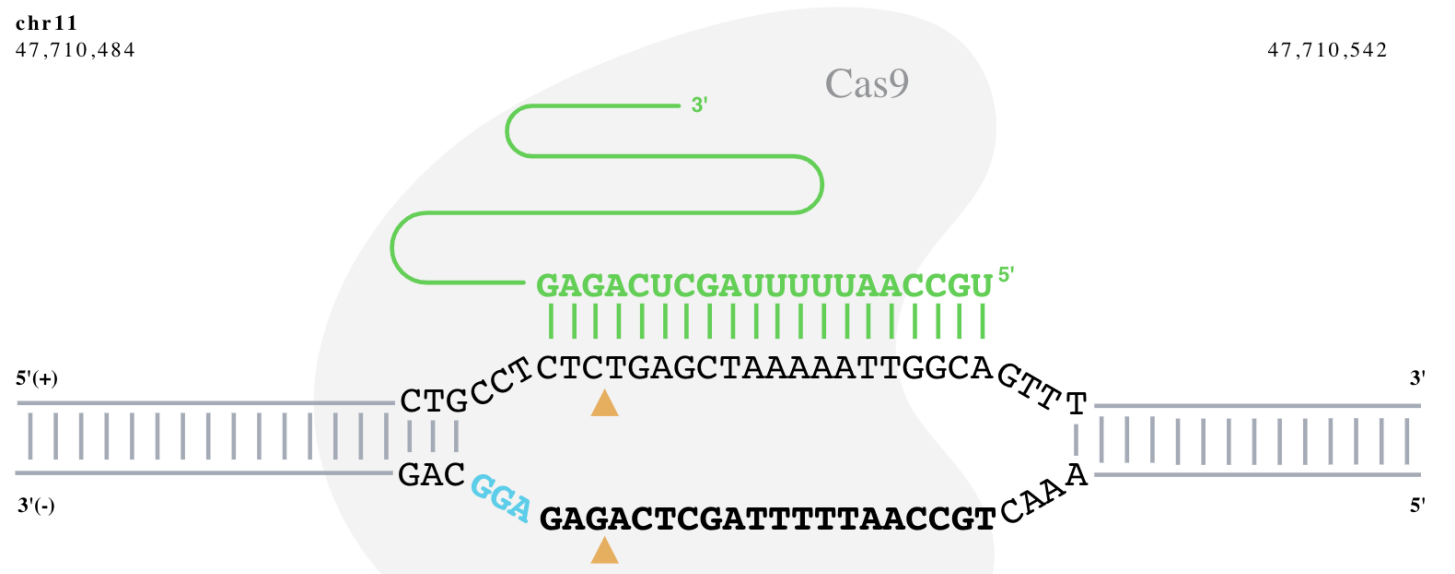

Figure 2. Top-ranked guide RNA sequence (shown in green) for the AGBL2 gene. Nucleotide sequence in light blue represents the PAM region. Orange triangles show the cut site. DNA segment in bold represents the target sequence (Synthego, 2021b).

In the case of the SUCLA2 gene, which has a transcript Ensembl identifier of ENST00000378654, the topranked guide RNA had the sequence CCCAGAACCUAGAAAGAUUG, which targeted the sequence CCCAGAACCTAGAAAGATTG, located in the thirteenth human chromosome. This target sequence is located in an exon that results from most transcripts, making this guide RNA the most convenient possible for knocking out this gene. As shown in Figure 3, here the Cas9 ribonucleoprotein will bind to the antisense strand of the selected gene, generating a double-stranded break at the 47,997,033 location and having GGG as its PAM region.

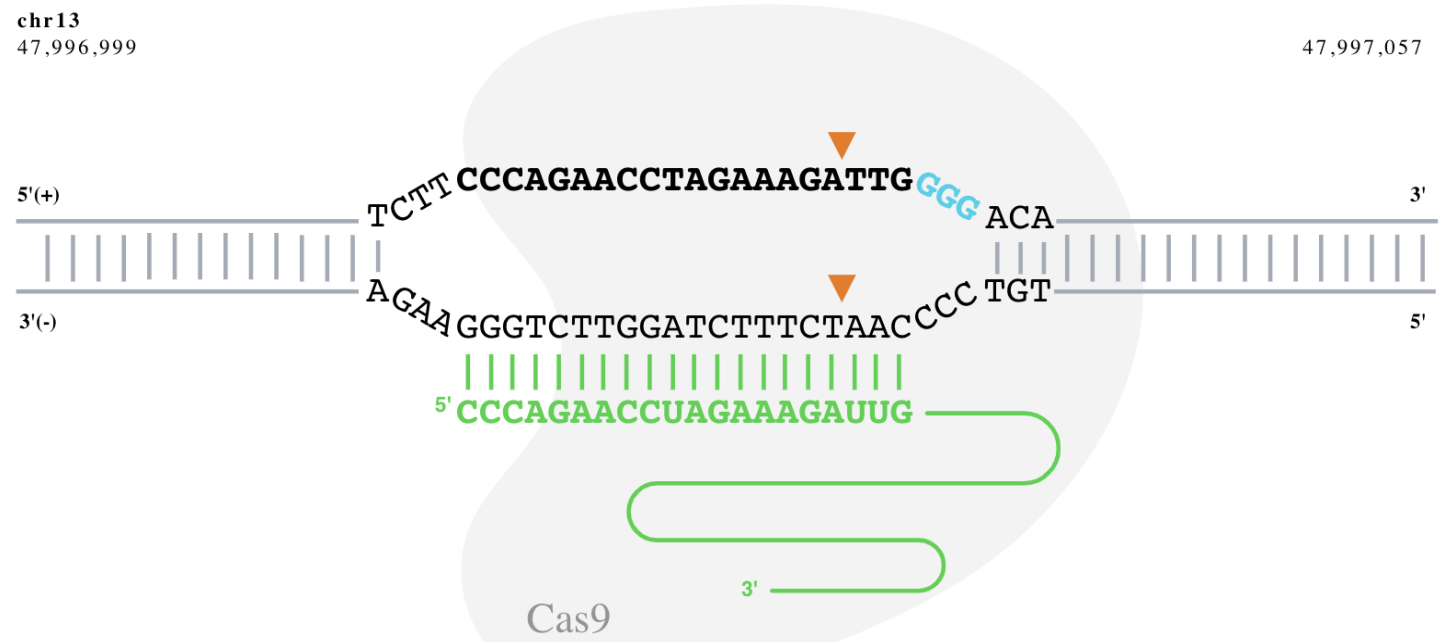


Figure 3. Top-ranked guide RNA sequence (shown in green) for the SUCLA2 gene. Nucleotide sequence in light blue represents the PAM region. Orange triangles show the cut site. DNA segment in bold represents the target sequence (Synthego, 2021c).

In the case of the SP110 gene, which has a transcript Ensembl identifier of ENST00000358662, the topranked guide RNA had the sequence UACAGGGAUCAAAUUUCUAC, which targeted the sequence TACAGGGATCAAATTTCTAC, located in the second human chromosome. This target sequence is located towards the start of the gene's coding sequence, plus it is located in an exon that results from most transcripts, making this guide RNA the most convenient possible for knocking out this gene. As shown in Figure 4, here the Cas9 ribonucleoprotein will bind to the antisense strand of the selected gene, generating a double-stranded break at the 230,215,100 location and having AGG as its PAM region.

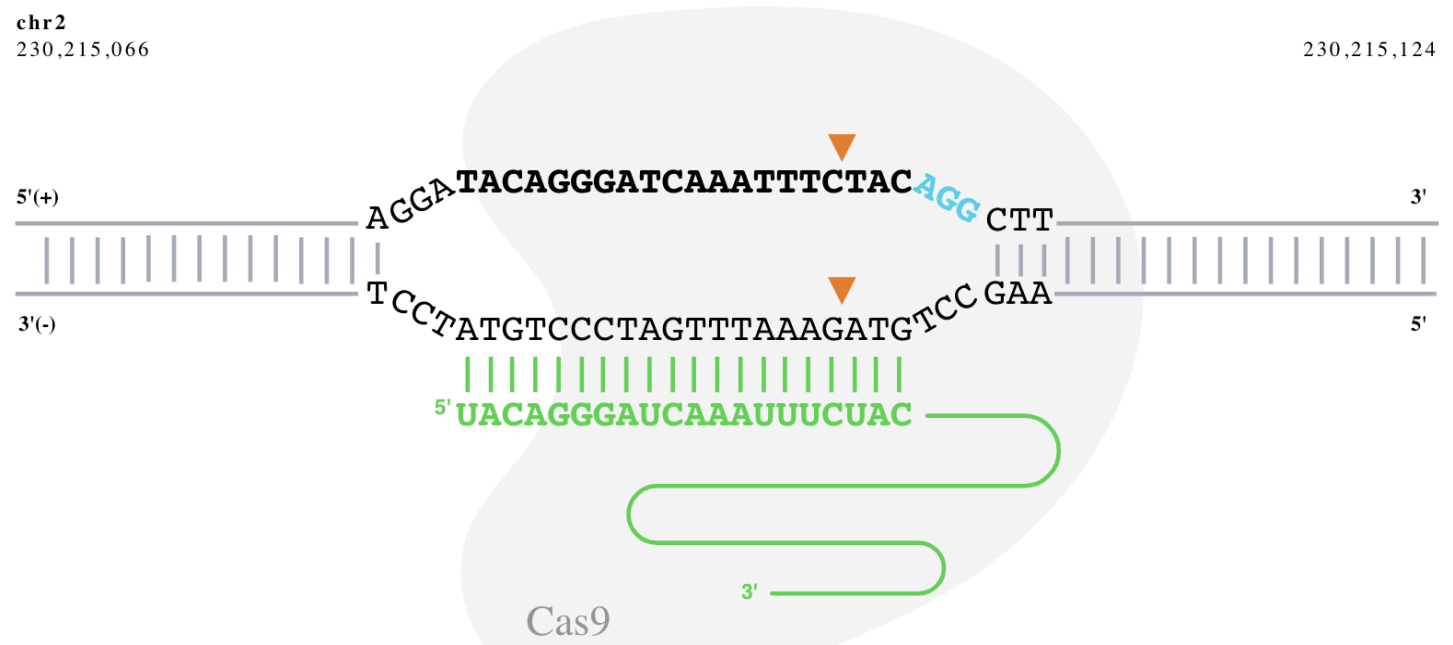

Figure 4. Top-ranked guide RNA sequence (shown in green) for the SP110 gene. Nucleotide sequence in light blue represents the PAM region. Orange triangles show the cut site. DNA segment in bold represents the target sequence (Synthego, 2021d).

The four models above show how CRISPR could be used in order to break the dsDNA molecule and, in turn, knock out genes, in this case the four suicide-related ones (APH1B, AGBL2, SUCLA2, and SP110). However, even though this technique can be a powerful tool for genome editing, it has one main limitation that is keeping it from being massively used in clinical procedures: off-targets. Off-targets occur when Cas9 binds to an unintended genomic site, leading to a cut in the wrong location and potentially causing mostly unknown side effects if there are more than three mismatches between target sequences and 20 nucleotides of guide RNA (Naeem et al., 2020).

Off-target effects may cause severe problems in the organism at the genomic level since they could lead to the alteration of a gene's function, which could result in genomic instability. Even though DNA follows the highly specific base pairing rules (adenine with thymine and cytosine with guanine), there is still the chance of off-targets to occur with three to five mismatches within the distal part of the PAM region, which refers to the short DNA sequence located around three to four nucleotides apart from the cut site that allows the Cas 9 protein to cleave the DNA molecule. 
Table 1. Off-targets found for each one of the suicide-related genes studied.

\begin{tabular}{|l|l|l|}
\hline Gene & gRNA sequence & Off-targets found \\
\hline APH1B & UCUUGCCAUGAACCAAACAA & $0,0,0,12,115$ \\
\hline AGBL2 & UGCCAAUUUUUAGCUCAGAG & $0,0,0,13,144$ \\
\hline SUCLA2 & CCCAGAACCUAGAAAGAUUG & $0,0,1,24,282$ \\
\hline SP110 & UACAGGGAUCAAAUUUCUAC & $0,0,0,16,98$ \\
\hline
\end{tabular}

In order to avoid severe off-target effects, it is crucial to choose the most convenient guide RNA to make the DNA cleavage as precise as possible. Synthego's guide RNA design tool, which was used for this paper's research, allows the selection of the guide RNA that would most likely result in the least number of off-targets among all the existing gRNAs for the selected gene. Regarding the suicide-related genes that were studied, the total off-targets found are summarized in Table 1. Based on the possibility of having negative side effects caused by off-targets when using CRISPR, guide RNAs with minimal off-targets were chosen for all four cases, providing results that, if applied in a real-life situation, would most likely be favorable.

\section{Conclusion}

This study demonstrates the effectiveness of CRISPR as a potential technique to reduce suicide risk and, in turn, make the number of suicide-related deaths decrease worldwide. By knocking out genes APH1B, AGBL2, SP110, and SUCLA2, the negative effects someone's genome may have on their risk to die by suicide can potentially decrease if CRISPR is done adequately. Nonetheless, in order to do so, it is crucial to choose the best guide RNAs so that the number of off-targets is minimized, helping reduce side effects (such as unintended mutations) as much as possible.

For the future scope of this research, scientists can apply this study in real life situations, that is, in a laboratory setting. However, it is important to point out that this genome editing technique is still trying to be proven as safe and feasible before being used in human beings, so ex vivo cell-based CRISPR could be performed alternatively. This could help prove the viability of this procedure and make it more convincing that, in the long run, genome editing techniques such as CRISPR could be even applied in psychiatry settings for people who suffer from suicidal thoughts or behaviors.

\section{Acknowledgments}

I would like to thank my teacher, Mr. Javier Cornejo, for his support and guidance throughout the process of completing this research project.

\section{References}

American Foundation for Suicide Prevention. (2019). Risk factors, protective factors, and warning signs. American Foundation for Suicide Prevention. https://afsp.org/risk-factors-protective-factors-and-warning-signs 
Bekris, L., Tsuang, D., Peskind, E., Yu, C., Montine, T., Zhang, J., Zabetian, C., \& Leverenz, J. (2015). Cerebrospinal fluid A $\beta 42$ levels and APP processing pathway genes in Parkinson's disease. Movement Disorders, 30(7), 936944. https://doi.org/10.1002/mds.26172

Bondy, B., Buettner, A., \& Zill, P. (2006). Genetics of suicide. Molecular Psychiatry, 11(4), 336-351. https://doi.org/10.1038/sj.mp.4001803

Centers for Disease Control and Prevention. (2021). Facts About Suicide. Centers for Disease Control and Prevention. https://www.cdc.gov/suicide/facts/index.html

Coon, H., Darlington, T., DiBlasi, E., Callor, B., Ferris, E., Fraser, A., Yu, Z., William, N., Das, S., Crowell, S., Chen, D., Anderson, J., Klein, M., Jerominski, L., Cannon, D., Shabalin, A., Docherty, A., Williams, M., Smith, K., $\&$ Keeshin, B. (2020). Genome-wide significant regions in 43 Utah high-risk families implicate multiple genes involved in risk for completed suicide. Molecular Psychiatry, 25, 3077-3090. https://doi.org/10.1038/s41380-018$\underline{0282-3}$

Doench, J. (2017). Am I ready for CRISPR? A user's guide to genetic screens. Nature Reviews Genetics, 19(2), 6780. https://doi.org/10.1038/nrg.2017.97

Dwivedi, Y. (2012). The Neurobiological Basis of Suicide. CRC Press.

Ibrahim, O., \& Umar, A. (2018). CRISPR Technology Advantages, Limitations and Future Direction. Journal of Biomedical and Pharmaceutical Sciences, 1(2). https://www.hilarispublisher.com/open-access/crispr-technologyadvantages-limitations-and-future-direction.pdf

Karaca, E., Harel, T., Pehlivan, D., Jhangiani, S., Gambin, T., Coban, Z., Gonzaga, C., Erdin, S., Bayram, Y., Campbell, I., Hunter, J., Atik, M., Van, H., Yuan, B., Wiszniewski, W., Isikay, S., Yesil, G., Yuregir, O., Tug, S., \& Aslan, H. (2015). Genes that Affect Brain Structure and Function Identified by Rare Variant Analyses of Mendelian Neurologic Disease. Neuron, 88(3), 499-513. https://doi.org/10.1016/j.neuron.2015.09.048

Leu, J., Chang, S., Mu, C., Chen, M., \& Yan, B. (2018). Functional domains of SP110 that modulate its transcriptional regulatory function and cellular translocation. Journal of Biomedical Science, 25(1).

https://doi.org/10.1186/s12929-018-0434-4

McGuffin, P., Marušič, A., \& Farmer, A. (2001). What Can Psychiatric Genetics Offer Suicidology? Crisis, 22(2), 61-65. https://doi.org/10.1027//0227-5910.22.2.61

Naeem, M., Majeed, S., Hoque, M., \& Ahmad, I. (2020). Latest Developed Strategies to Minimize the Off-Target Effects in CRISPR-Cas-Mediated Genome Editing. Cells, 9(7). https://doi.org/10.3390/cells9071608

Nowicki, S. (2017). Holt McDougal Biology. Houghton Mifflin Harcourt Publishing Company.

Pedersen, N., \& Fiske, A. (2010). Genetic influences on suicide and nonfatal suicidal behavior: Twin study findings. European Psychiatry, 25(5), 264-267. https://doi.org/10.1016/j.eurpsy.2009.12.008

Redman, M., King, A., Watson, C., \& King, D. (2016). What is CRISPR/Cas9? Archives of Disease in Childhood Education \& Practice Edition, 101(4), 213-215. https://doi.org/10.1136/archdischild-2016-310459 
Synthego. (2021a). Guide RNA for AGBL2 gene. Synthego. https://design.synthego.com/\#/validate/results?genome $=$ homo sapiens gencode 26 primary \&nuclease $=$ cas $9 \&$ guide $=$ UGCCAAUUUUUAGCUCAGAG

Synthego. (2021b). Guide RNA for APHIB gene. Synthego. https://design.synthego.com/\#/validate/results?genome $=$ homo sapiens gencode 26 primary $\&$ nuclease $=$ cas 9 \& guide=UCUUGCCAUGAACCAAACAA

Synthego. (2021c). Guide RNA for SP110 gene. Synthego. https://design.synthego.com/\#/validate/results?genome=homo sapiens gencode 26 primary \&nuclease $=$ cas $9 \&$ guide $=$ UACAGGGAUCAAAUUUCUAC

Synthego. (2021d). Guide RNA for SUCLA2 gene. Synthego. https://design.synthego.com/\#/validate/results?genome $=$ homo sapiens gencode 26 primary \&nuclease $=$ cas $9 \&$ guide $=$ CCCAGAACCUAGAAAGAUUG

Völgyi, K., Gulyássy, P., Háden, K., Kis, V., Badics, K., Kékesi, K., Simor, A., Györffy, B., Tóth, E., Lubec, G., Juhász, G., \& Dobolyi, A. (2015). Synaptic mitochondria: A brain mitochondria cluster with a specific proteome. Journal of Proteomics, 120, 142-157. https://doi.org/10.1016/j.jprot.2015.03.005

World Health Organization. (2021). Suicide. World Health Organization. https://www.who.int/news-room/factsheets/detail/suicide 